\title{
Domain Structures in Co/Pd Multilayers
}

\author{
V. Kamberský, P. de Haan, J.C. Lodder, J. Šimšová ${ }^{1}$, R. Gemperle ${ }^{1}$ \\ MESA, University of Twente, P.O. Box 217, 7500 AE Enschede \\ ${ }^{1}$ Institute of Physics, Czech Acad.Sci,,Cukrovarnická 10,16200 Prague
}

\begin{abstract}
Domain observations and measurements of mean domain sizes in $\mathrm{Co} / \mathrm{Pd}$ multilayers with high perpendicular anisotropy are reported and compared with the predictions of the stacked-stripe model of domain structure. The estimated high wall energies are consistent with measured anisotropy. Properties of the model in the thin-layer limit are discussed.
\end{abstract}

\section{INTRODUCTION}

Potential application of $\mathrm{Co} / \mathrm{Pd}$ and $\mathrm{Co} / \mathrm{Pt}$ multilayers in magneto-optical recording draws attention to films with large perpendicular anisotropy, coercivity and remanence. Inferences about the domain structure of "hard" films are mostly based on comparison [1-5] of measured hysteresis loops with predictions of the stripe-domain model extended to multilayers $[1,2]$, although direct domain observations $[4,6]$ and measurements [7] have recently been reported.

We report direct measurements of domain sizes in "hard" Co/Pd multilayers prepared by if sputtering [8] and compare them with the model predictions. A comment is added on the similarity of these predictions to those based on averaged uniform material parameters, noticed in the behaviour of theoretical hysteresis loops [4].

\section{MODEL CALCULATION}

In the periodic-stripe model extended to multilayers $[1,2]$ (Fig. 1) the magnetostatic energy $E_{d}$ is computed as a function of the domain width, $D$. The formulation [2] allows to evaluate the left-hand side of the force-balance equation

$$
F_{m} \equiv D \partial e_{m} / \partial D=\sigma / D
$$

where $e_{m}=E_{d} / V_{m}$ is scaled to the magnetic $(C o)$ volume, $V_{m}, \sigma$ is the wall energy density and $F_{m}$ has the meaning of magnetostatic pressure (force per unit wall area).

Fig. 2 is a scaled plot of this quantity in the (presently) relevant range of model parameters: $D$ is the domain width, $T$ is the total film thickness, $f=V_{m} / V_{f}$ is the filling factor $\left(V_{f}\right.$ is the total film volume), and $\mathrm{N}$ is the number of bilayers.

Selecting scales for this plot, we find that

$$
F_{f 0}=f^{-1} F_{m} /\left(0.5 \mu_{0} M_{s}^{2}\right)
$$

(where $\mathrm{M}_{\mathrm{s}}$ pertains to magnetic layers), $v s . T / D$ gives curves which only little depend on the stacking parameter $\mathrm{N}$ and $f$. This feature is discussed in sect.IV.

Manuscript received February 15,1993 . This research is supported by the EC CAMST program

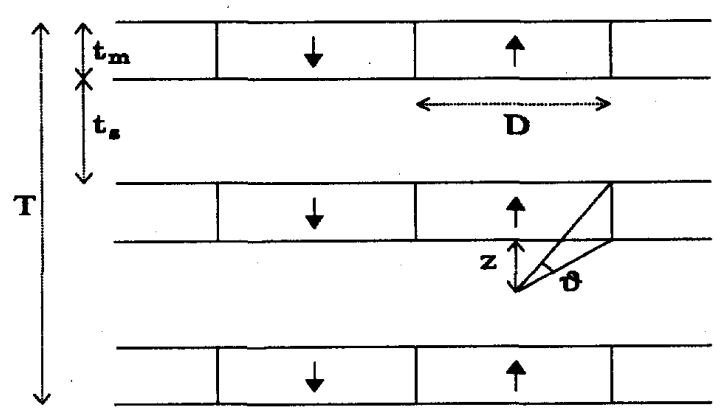

Figure 1 The periodic stack-stripe domain model

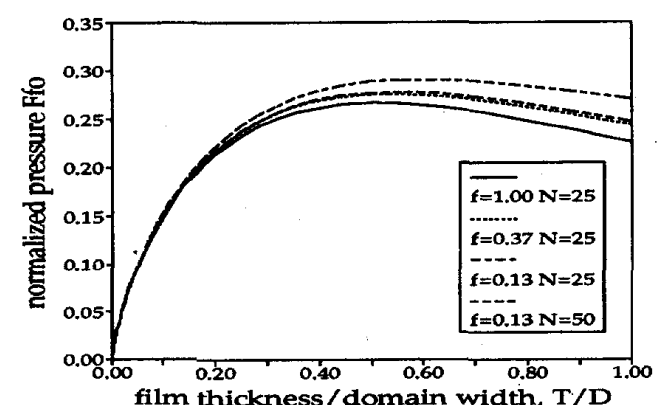

Fig. 2 Normalized wall-pressure $F_{f 0}$ (2) vs. film thickness-to-domain width ratio.

From (1) and Fig. 2 the wall energy may be determined if the equilibrium $D$ is estimated experimentally.

\section{EXPERIMENT}

Magnetic properties of a series of $\mathrm{Co} / \mathrm{Pd}$ multilayers prepared by if sputtering in $\mathrm{Ar}$ have been reported previously [8]. The following table summarizes parameters of the samples used in the present domain study.

TABLE I

\begin{tabular}{|c|c|c|c|c|c|c|}
\hline & nm & $\begin{array}{r}T \\
\mathrm{~nm} \\
\end{array}$ & f & $\begin{array}{c}\mathrm{M}_{\mathrm{e}} \\
\mathrm{MAm}^{-1}\end{array}$ & $\begin{array}{c}\mathrm{H}_{r} \\
\mathrm{kAm}^{-1} \\
\end{array}$ & $\begin{array}{c}\mathrm{K}_{11} \\
\mathrm{MJm}^{-3} \\
\end{array}$ \\
\hline a & 0.85 & 57.4 & 0.37 & 1.85 & 36 & 2.5 \\
\hline b & 0.43 & 83.9 & 0.13 & 1.6 & 111 & 3.2 \\
\hline
\end{tabular}

THICKNESS AND MAGNETIC PROPERTIES OF SAMPLES a AND $b(\mathrm{~N}=25)$ 


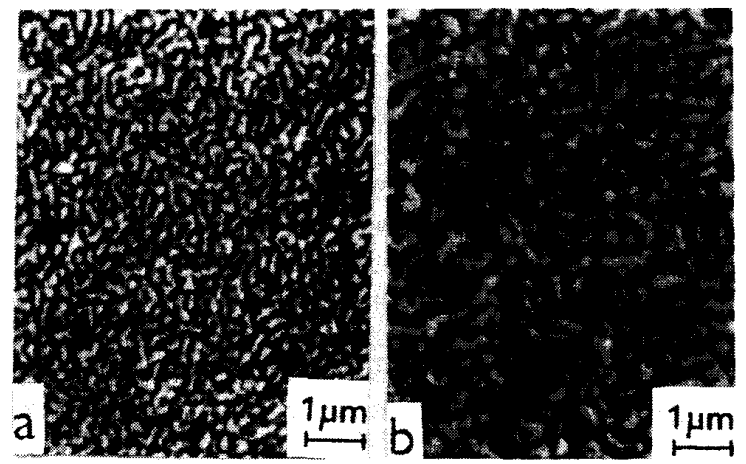

Fig. 3 Colloid-SEM images of domain structure of samples $a$ and $b$.

We used the CSEM method (dried colloid Bitter patterns observed by scanning electron microscopy [9]) to obtain images of the domain structure in various states of film magnetization. Figs. $3 a, b$ show the domain structures on the two samples, decorated after saturation followed by several cycles on minor loops in the field range $\pm 160 \mathrm{kA} / \mathrm{m}$. In accordance with our previous experience on medium-coercivity $\mathrm{CoCr}$ films [10], the domain widths in such states are close to those obtained by ac demagnetization. This was also verified on our sample $a$. The average transverse domain dimensions $(D)$ obtained statistically on samples $a$ and $b$ are $90 \mathrm{~nm}$ and $145 \mathrm{~nm}$, respectively.

\section{DISCUSSION}

\section{A. Scaling of magnetostatic forces.}

The normalized pressure $F_{\text {fo }}(2)$ plotted in Fig.2 is a filmaveraged quantity: it may be written as

$$
\mathrm{F}_{\mathrm{f} 0}=\left(\mathrm{D} \partial \mathrm{e}_{\mathrm{f}} / \partial \mathrm{D}\right) /\left(0.5 \mu_{0} \mathrm{M}_{\mathrm{f}}^{2}\right)
$$

with $e_{f}=E_{d} / V_{f}$ and $M_{f}=f M_{s}$ scaled to the total film volume. This pressure is formally balanced by the average surface energy $\sigma_{\mathrm{f}}=\mathrm{f} \sigma$ in the geometrical stack-domain boundary. The balance (1) may be written in two "normalized" forms, $\mathrm{F}_{\mathrm{m} 0}=\mathrm{L}_{\mathrm{m}} / D$ or $\mathrm{F}_{\mathrm{f} 0}=\mathrm{L}_{\mathrm{f}} / D$, where $\mathrm{L}_{m}=\sigma /\left(0.5 \mu \mathrm{M}_{\mathrm{s}}{ }^{2}\right)$ and $\mathrm{L}_{\mathrm{f}}=$ $\sigma_{\mathrm{f}} /\left(0.5 \mu \mathrm{M}_{\mathrm{f}}^{2}\right)$, resp., denote the "material lengths" of the magnetic layers and of the multilayer as a whole. The second viewpoint is assumed by Hashimoto et al. [11] in crude estimates of the expected domain dimensions.

Figs. 2 and $4 \mathrm{a}$ indicate that, indeed, in the "thin-flat" limit $\left(\mathrm{t}_{\mathrm{m}}<<\mathrm{D}\right), \mathrm{F}_{\mathrm{fo}}$ and hence also $\mathrm{L}_{\mathrm{f}}$ are appropriate chacteristics: the equilibrium domain sizes can be approximately obtained in terms of these average parameters (ignoring the actual geometrical alignment of individual layers) from single-layer theory [12].This is confirmed by the common scaling at the "thin" end of Fig.4b which shows the equilibrium domain size vs. total film thickness, both normalized to $\mathrm{L}_{\mathrm{f}}$.

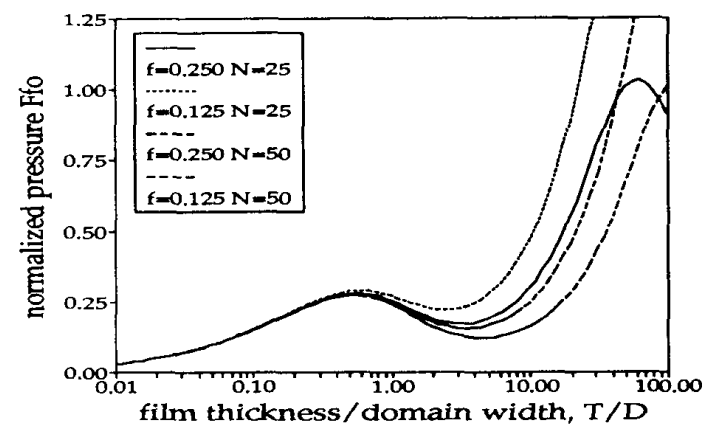

Fig.4a Normalized wall pressure $\mathrm{F}_{n} v s$. film thickness-to-domain width ratio.

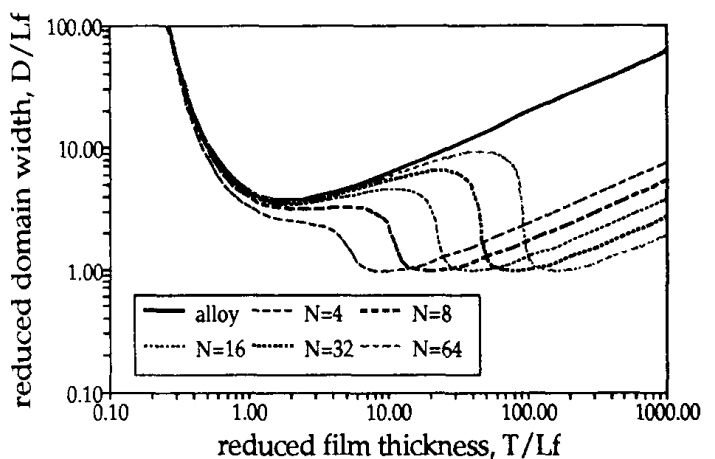

Fig. 4b Reduced domain width $v s$. reduced film thickness ( $\mathrm{f}=0.25$ ).

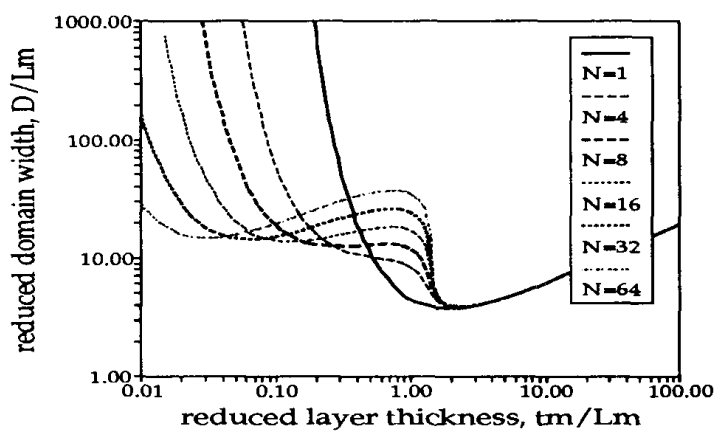

Fig. 5 Reduced domain width vs. magnetic layer thickness $(\mathrm{f}=0.25$ )

The sharp departure from the "average" scaling in these plots is connected with keeping constant $\mathrm{f}$ and $\mathrm{N}$ on individual curves, which corresponds to $\mathrm{T}$ increased by proportional "inflation" of the magnetic $\left(t_{m}\right)$ and spacer $\left(t_{s}\right)$ layers. The $\mathrm{D}$ vs. $\mathrm{T}$ plots have only one minimum if $\mathrm{T}$ increases by "stacking" $[2,4,7]$ ( $\mathrm{T}$ proportional to $\mathrm{N}$ at constant $t_{m}$ and $f:$ read $D$ values on verticals of given $t_{m} / L_{m}$ or of $\mathrm{T} / \mathrm{L}_{\mathrm{f}}=\mathrm{Nt}_{\mathrm{m}} / \mathrm{L}_{\mathrm{m}}$ in Figs.5 and $4 \mathrm{~b}$, resp.).

Appropriate scaling of these "inflation" plots at high T/D is in terms of magnetic-layer parameters, $t_{m}$ and $F_{m}\left(M_{s}\right.$ and $\mathrm{L}_{\mathrm{m}}$ ), as shown in Fig.5. The high- $\mathrm{t}_{\mathrm{m}} / \mathrm{D}$ end is the Kittel limit 


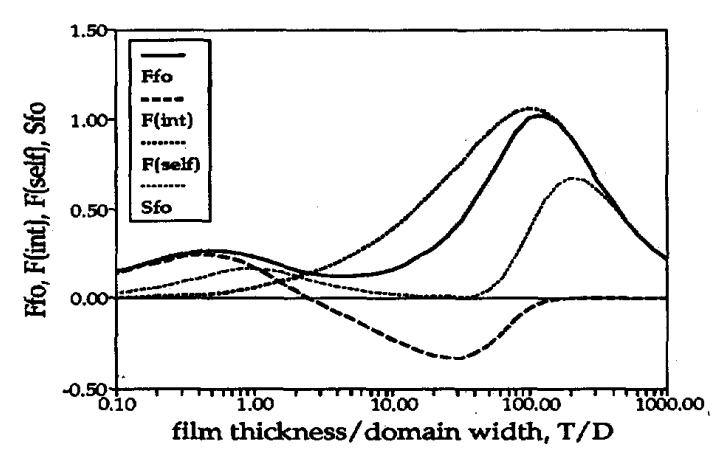

Fig.6 Norm.pressure components and derivative $S_{f 0}$ (see text; $f=0.25, N=50$ )

$[13,12]$ where dipolar fields between laterally neighbouring domains are confined to individual layers.

On the other hand, the intuitively obvious reason for the "alloy-analogy" in domain behaviour on the "thin" end is the penetration of stray-fields of flat domains across the whole film thickness (so that all dipoles effectively interact with each other). This is illustrated in Fig.6 where the part of $F_{f 0}$ arising from interaction between different layers, $F($ int), is separated from the derivatives of the self-energy of individual layers, $F$ (self). If $t_{m}<D$, the inter-layer force accounts for practically all the magneto-static pressure which generates the domains; and the observed narrow domains can only be sustained magnetostatically if they penetrate the whole film.

The transition between relatively strong and weak interlayer force occurs in a narrow range of magnetic and spacer thickness; plots analogous to Figs.4, 5 and 6 show a critical range at $\left.\sqrt{\left(t_{m}\right.} t_{s}\right) \approx 3 L_{m}$, and a relation between the bilayer thickness and the domain size in this range,

$$
t_{m}+t_{s} \approx 0.6 D \text {. }
$$

The weak line in Fig.6 is a plot of $S_{f 0}=\left[F_{f 0}+D \partial F_{f 0} / \partial D\right] / 2$ which is proportional to $D^{2} \partial^{2}\left(E_{d}+E_{\text {wall }}\right) / \partial D^{2}$ and thus measures stability of the model structure; its deep minima in the range (4) are close to the (negative) minima of F(int).

The relation (4) and the "resonant" feature in the interlayer pressure (Fig.6) allow an intuitive interpretation based on local estimates of the stray field of a single thin flat domain (Fig.1): the field at a distance $z$ below its centre is positive (parallel to $M$ ) and equal to $2 \mu_{0} M_{s} \vartheta / \pi$, where (for $\left.t_{m}<<z, D\right) \quad \vartheta \approx(2 t / D) /\left(1+4 z^{2} / D^{2}\right)$ indicates effective penetration to $\mathrm{z}_{\mathrm{o}} \approx D / 2$. At such (fixed) distance, the angle $\vartheta$ and hence the positive field decreases with either increasing or diminishing $D$, which explains the changing sign of the energy derivative (negative inter-layer pressure) in Fig.6.

\section{B. Estimated domain wall energy.}

Comparing the observed domain sizes with the predictions of the stripe model, we estimate the "material length" $L_{m}$ (denoted $\tau$ in recent model studies $[1,2,5,7]), L_{m}=D F_{m 0}=$ f D F $F_{f 0}$ and the wall energy density: $L_{m}=9.2$ and $5.5 \mathrm{~nm}$, $\sigma \approx 20$ and $9 \mathrm{~mJ} / \mathrm{m}^{2}$, for samples $a$ and $b$, respectively. The higher value corresponds well to the expected wall energy, $\sigma=4 \sqrt{ }\left(A K_{u}\right)$ with the measured anisotropy constant $K_{u}$ and the value of exchange constant $A$ for bulk Co $(\approx 10 \mathrm{pJ} / \mathrm{m})$. The lower values obtained for sample $b$ with lower Co thickness are closer to those reported by Zeper et al. [5] and Donnet et al.[7], corresponding to "effective" exchange stiffness of only $20 \%$ of the bulk value. This may be easily ascribed to deteriorating lateral coherence of very thin $\mathrm{Co}_{0}$ films in sputtered multilayers, with columnar structure. Preliminary measurement on other "hard" samples with small $t_{m}\left(H_{c}=96\right.$ and $\left.152 \mathrm{kA} / \mathrm{m}\right)$ leads, however, to $L_{m}=6$ to $8 \mathrm{~nm}$, and detailed investigation of the differences in domain structure and morphology is desirable.

\section{CONCLUSIONS}

Observation of the domain structure and measurement of domain sizes on minor hysteresis loops of magnetically "hard" $\mathrm{Co} / \mathrm{Pd}$ multilayers (combined with previously measured magnetic parameters) shows good and moderate agreement with predictions of the stripe-domain model [2] in the case of medium and high coercitivity films, respectively; possible correlation with structural properties deserves further investigation.

Formal results of model calculations are discussed: the analogy between domain patterns expected in thin multilayers and in films of corresponding alloys follows from prevalence of inter-layer magnetostatic forces; rather sharp transitions to significantly denser domain patterns can be expected with increasing bilayer thickness.

\section{REFERENCES}

[1] A. Suna Joumal of Applied Physics 59, p313, (1986)

[2] H.J.G. Draaisma, WJ.M de Jonge Journal of Applied Physics 62 p 3318 (1987)

[3] W.R. Bennet, C.D. England, D.C. Person, C.M. Falco Journal of Applied Physics 69 p4384 (1991)

[4] S. Honda, Y. Ikegawa, T. Kusiuda J. Mag. Mag. Mat. 111 p 273 (1992)

[5] W.P Zeper, H.W. van Kesteren, B.AJ. Jacobs, J.H.M. Spruit, P.F. Carcia Journal of Applied Physics 70 p 2264 (1991)

[6] E.T.M. Lacey, PJ. Grundy IEEE Transaction on Magnetics 26 p 2356 (1990)

[7] D.M. Donnet, J.N. Chapman, H.W. van Kesteren, W.B. Zeper J. Mag. Mag. Mat. 115 p342 (1992)

[8] P. de Haan, Q. Meng, T. Katayama, J.C. Lodder J. Mag. Mag. Mat 113 p29 (1992)

[9] J. Simsovd, R.Gemperle, J.C. Lodder J. Mag. Mag. Mat. 95 p85 (1991)

[10] J. Simsovd, V. Kamberský, R.Gemperle, J.C. Lodder, WJ.M.A. Geerts, B. Otter, P. ten Berge J. Mag. Mag. Mat. 101 p196 (1991)

[11] S. Hashimoto, Y. Ochiai,J. Mag. Mag. Mat. 88 p.211 (1990)

[12] Z. MAlek, V. Kambersky, Czech.J.Phys. 8 p.416 (1958)

[13] C. Kittel, Phys. Rev. 70 p.965 (1946) 